\title{
Gauss-Bonnet dark energy by Lagrange multipliers
}

\author{
Salvatore Capozziello ${ }^{1,2}$, Andrey N. Makarenko ${ }^{4}$ and Sergei D. Odintsov ${ }^{3,4,5}$ \\ ${ }^{1}$ Dipartimento di Scienze Fisiche, Università di Napoli "Federico II", Napoli, Italy \\ ${ }^{2}$ INFN Sez. di Napoli, Compl. Univ. di Monte S. Angelo, Edificio G, Via Cinthia, I-80126, Napoli, Italy, \\ ${ }^{3}$ Instituciò Catalana de Recerca $i$ Estudis Avançats (ICREA) and Institut de Ciencies de l'Espai (IEEC-CSIC), \\ Facultad de Ciencies, C5, Campus UAB, 08193 Barcelona, Spain \\ 4 Tomsk State Pedagogical University, Tomsk, Russia \\ 5 Eurasian National University, Astana, Kazakhstan
}

(Dated: October 4, 2018)

\begin{abstract}
A string-inspired effective theory of gravity, containing Gauss-Bonnet invariant interacting with a scalar field, is considered in view of obtaining cosmological dark energy solutions. A Lagrange multiplier is inserted into the action in order to achieve the cosmological reconstruction by selecting suitable forms of couplings and potentials. Several cosmological exact solutions (including dark energy of quintessence, phantom or Little Rip type) are derived in presence and in absence of the Lagrange multiplier showing the difference in the two dynamical approaches. In the models that we consider, the Lagrange multiplier behaves as a sort of dust fluid that realizes the transitions between matter dominated and dark energy epochs. The relation between Lagrange multipliers and Noether symmetries is discussed.
\end{abstract}

PACS numbers: 04.50.Kd, 04.20.Jb, 04.20.Cv, 98.80.Jk

\section{INTRODUCTION}

Astrophysical data indicate that the observed universe is in an accelerated phase [1]. This acceleration is induced by the so-called dark energy (see Ref. [2] for a recent review and references therein) which nature and properties are not yet understood at fundamental level. In the most theoretical models considered in the literature, the dark energy is constituted by some ideal fluid with a specific equation of state (EoS) sometimes exhibiting non-standard properties like negative pressure and/or a negative entropy. On the other hand, dark energy can be considered as a global phenomenon associated with modifications of gravity [3]. In fact, its presence could point out that Einstein's General Relativity cannot be retained as the final theory of gravitational interaction at cosmological scales. In this sense, General Relativity presents problems at UV (Quantum Gravity) and IR (cosmology) scales [4]. A similar situation appear for dark matter phenomena. No fundamental candidate has been revealed up to now and dynamics of self-gravitating structures could be addressed by modifications of gravity [5].

A further problem is that it is not clear why dark energy had no effect at early epochs while it gives dominant contribution in today observed universe. According to the latest observational data, dark energy currently accounts for about $73 \%$ of the total mass-energy amount of the universe (see, for example, Ref. [6]).

The main feature of dark energy is that its EoS parameter $w_{\mathrm{D}}$ is negative:

$$
w_{\mathrm{D}}=p_{\mathrm{D}} / \rho_{\mathrm{D}}<0,
$$

where $\rho_{\mathrm{D}}$ is the dark energy density and $p_{\mathrm{D}}$ the pressure. According to the standard cosmological model, this property gives rise to the reported apparent acceleration of the
Hubble fluid. Although current data favor the standard $\Lambda \mathrm{CDM}$ cosmology, the uncertainties in the determination of the EoS dark energy parameter $w$ are still too large, namely $w=-1.04_{-0.10}^{+0.09}$. Hence, one is not able to determine, without doubt, which of the three cases: $w<-1$, $w=-1$, or $w>-1$ is the one actually realized in our universe [7, 8]. Future observations should better constrain this range of values giving also indications on the nature of dark energy [9].

In order to explain dark energy at very fundamental level, string/M-theory could suggest reliable effective models to be compared with observations. In particular, further gravitational terms, emerging from this theory, could become important at current, low-curvature universe (being not essential at intermediate epochs from strong to low curvature). For instance, in the study of string-inspired gravity near the initial singularity, the role of the Gauss-Bonnet (GB) topological term, coupled with scalar potentials, is important for the occurrence of non-singular cosmology [10, 11]. The dilaton coupled to higher-order curvature corrections, for example, assumes an important role near the initial singularity, as discussed in [12]. Furthermore, string-inspired gravity with Gauss-Bonnet term interacting with a scalar field has been proposed as a realistic candidate to address the dark energy issue [13]. Specifically, as it was shown in Ref.[13], Gauss-Bonnet dark energy may lead to the occurrence of phantom cosmology without ghosts: in this case, the dilaton is a canonical scalar. Further aspects of Gauss-Bonnet accelerating cosmology have been discussed in detail in [14, 15].

Recently, a new dark energy model has been proposed [16, 17]. It consists in considering two scalars where one of them is given by a Lagrange multiplier. This multiplier puts a natural constraint on the form on the second scalar field (in particular on its coupling and self-interacting po- 
tential) allowing that the emerging dark energy behavior evolves in a dust-matter dominated era, as requested by observations going back in the redshift $z$. The interesting feature of this approach is that the whole system contains a single dynamical degree of freedom and this fact allows to solve several shortcomings related to the fine-tuning of $\Lambda \mathrm{CDM}$ model, among them the cosmological constant problem 23. It is important to stress that such a Lagrange multiplier technique can be related to the existence of Noether symmetries and then it is a general approach to reduce dynamical systems and find out exact solutions, as we will discuss below [18].

The extension of $f(R)$ gravity via the addition of a Lagrange multiplier constraint has been proposed in Ref.[19]. Such model can be considered as a new version of modified gravity because dynamics and cosmological solutions are different from the standard version of $f(R)$ gravity without such constraint. This result is clear from a dynamical viewpoint: Lagrange multipliers are anholonomic constraints capable of reducing dynamics [20, 21]. Furthermore, using the Lagrange multiplier approach helps in the formulation of covariant renormalizable gravity [22].

In the present paper, we study the Gauss-Bonnet gravity with Lagrange multiplier constraints in view to recover realistic dark energy behaviors. Technically, we are considering a scalar-tensor-higher-order gravity where a Lagrange multiplier is considered. We explicitly show that it is possible to derive new cosmological solutions in this context. In particular, we compare the accelerating solutions in string-inspired Gauss-Bonnet gravity with and without the Lagrange multiplier term. It is demonstrated that, in the version with Lagrange multiplier, one gets large number of new accelerating cosmologies, including the phantom cosmologies where the dilaton kinetic term is canonical. As a more interesting example, the so-called Little Rip cosmology can be recovered.

The plan of the paper is the following. In Sect. II, we discuss the scalar Gauss-Bonnet gravity in presence of a Lagrange multiplier. In particular, we consider the Friedmann-Robertson-Walker (FRW) cosmology and derive exact solutions according to the choice of the scalar field $\varphi$. The general scheme of cosmological reconstruction of scalar Gauss-Bonnet gravity with Lagrange multiplier is pursued in Sect. III. Here we work out the whole method starting from the cosmological equations up to the final cosmological solutions. In Sec. IV, we rewrite the cosmological Friedmann equations as an autonomous system of first order differential equations and study its critical points. Sect. V is devoted to the summary and discussion of the results. The general discussion of the Lagrange multiplier method in view of the Noether Symmetry Approach is reported in Appendix A.

\section{SCALAR GAUSS-BONNET GRAVITY WITH LAGRANGE MULTIPLIER}

Let us study accelerating cosmology in string-inspired scalar Gauss-Bonnet gravity with Lagrange multiplier. To this end, a suitable action has the following form:

$$
\begin{aligned}
S & =\int d^{4} x \sqrt{-g}\left\{\frac{R}{2 \kappa^{2}}-\frac{\omega(\varphi)}{2} \partial_{\mu} \varphi \partial^{\mu} \varphi-V(\varphi)-\right. \\
& \left.-\varepsilon(\varphi) G-\lambda\left(\frac{1}{2} \partial_{\mu} \varphi \partial^{\mu} \varphi+U(\varphi)\right)\right\} .
\end{aligned}
$$

Here $\lambda$ is the Lagrange multiplier scalar, $\varepsilon, \omega$ and $U$ are arbitrary scalar functions and $G$ is Gauss-Bonnet invariant is

$$
G=R_{\mu \nu \alpha \beta} R^{\mu \nu \alpha \beta}-4 R_{\mu \nu} R^{\mu \nu}+R^{2} .
$$

The above effective action represents a string-inspired gravity which has been mainly considered for exponential potentials and without last term (for a single scalar). The interpretation of above action is related to specific compactification of superstring theory in four dimensions where, apart from the dilaton scalar field, one more scalar (given by the Lagrange multiplier) is considered.

The gravitational field equations are derived by varying with respect to the metric and assume the following form:

$$
\begin{aligned}
& \frac{1}{2 \kappa^{2}}\left(R_{\mu \nu}-\frac{1}{2} g_{\mu \nu} R\right)=-\frac{g_{\mu \nu}}{2}\left\{\frac{\omega(\varphi)}{2} \partial_{\rho} \varphi \partial^{\rho} \varphi+\right. \\
& \left.+V(\varphi)+\lambda\left(\frac{1}{2} \partial_{\rho} \varphi \partial^{\rho} \varphi+U(\varphi)\right)\right\}+ \\
& +\frac{\omega(\varphi)+\lambda}{2} \partial_{\mu} \varphi \partial_{\nu} \varphi+4\left(R_{\mu \nu}-\frac{1}{2} R g_{\mu \nu}\right) \square \varepsilon(\varphi)+ \\
& +4\left(R_{\mu}^{\alpha \beta}{ }_{\nu}-R^{\alpha \beta} g_{\mu \nu}\right) \nabla_{\alpha} \nabla_{\beta} \varepsilon(\varphi)-8 R_{\mu}^{\alpha} \nabla_{\alpha} \nabla_{\nu} \varepsilon(\varphi)+ \\
& +2 R \nabla_{\mu} \nabla_{\nu} \varepsilon(\varphi) .
\end{aligned}
$$

Let us now consider a FRW metric with flat spatial part:

$$
d s^{2}=-d t^{2}+a(t)^{2} \sum_{i=1,2,3}\left(d x^{i}\right)^{2} .
$$

Then by the variation over $\lambda$, we obtain the further equation

$$
0=\frac{\dot{\varphi}^{2}}{2}-U(\varphi)
$$

The cosmological Friedmann equations are then

$$
\begin{aligned}
\frac{3}{\kappa^{2}} H^{2} & =\frac{\omega(\varphi)+\lambda}{2} \dot{\varphi}^{2}+V(\varphi)+\lambda U(\varphi)+24 H^{3} \frac{d \varepsilon}{d t}= \\
& =(\omega(\varphi)+2 \lambda) U(\varphi)+V(\varphi)+24 H^{3} \frac{d \varepsilon}{d t},
\end{aligned}
$$




$$
\begin{aligned}
& -\frac{1}{\kappa^{2}}\left(2 \dot{H}+3 H^{2}\right)=\frac{\omega(\varphi)+\lambda}{2} \dot{\varphi}^{2}-V(\varphi)- \\
& -\lambda U(\varphi)-8 H^{2} \frac{d^{2} \varepsilon}{d t^{2}}-16 H \dot{H} \frac{d \varepsilon}{d t}-16 H^{3} \frac{d \varepsilon}{d t}= \\
& =\omega(\varphi) U(\varphi)-V(\varphi)-8 H^{2} \frac{d^{2} \varepsilon}{d t^{2}}-16 H \frac{d \varepsilon}{d t}\left(\dot{H}+H^{2}\right) .
\end{aligned}
$$

It is straightforward to derive, from the above equations, the effective EoS-parameter $w$ as:

$$
w=\frac{\omega(\varphi) U(\varphi)-V(\varphi)-8 H^{2} \frac{d^{2} \varepsilon}{d t^{2}}-16 H \frac{d \varepsilon}{d t}\left(\dot{H}+H^{2}\right)}{(\omega(\varphi)+2 \lambda) U(\varphi)+V(\varphi)+24 H^{3} \frac{d \varepsilon}{d t}} .
$$

The equation obtained by varying the action over the scalar field $\varphi$ (the Klein-Gordon equation) is a consequence of second Friedmann Eq.(8) and then is easily achieved.

Eqs.(7.8) can be easily solved. Indeed, there are unknown functions: $H, V, \varphi, \lambda, \omega$ and $\varepsilon$ which may be used to satisfy two equations. Therefore, if we want to obtain a closed system of equations, we must fix some of the functions $V, \lambda, \omega$ and $\varepsilon$ or select the field $\varphi$ and metric $a$, as well as two of the functions $V, \lambda, \omega$ and $\varepsilon$. For example, one can select:

$$
\omega(\varphi)=1, \quad U(\varphi)=\frac{m^{4}}{2} .
$$

Here $m$ is a constant with the dimension of mass. Also, the canonical scalar is considered. Then Eq. (6) gives

$$
\varphi=m^{2} t
$$

In this case, one can express the potential $V$ and the Lagrange multiplier $\lambda$ in terms of the Hubble rate $H$ and the function $\varepsilon$ :

$$
\begin{aligned}
V & =\frac{m^{4}}{2}+\frac{2 \dot{H}}{\kappa^{2}}-16 H \dot{\varepsilon}\left(\dot{H}+H^{2}\right)+H^{2}\left(\frac{3}{\kappa^{2}}-8 \ddot{\varepsilon}\right), \\
\lambda & =-1+\frac{8 \kappa^{2} H\left(\dot{\varepsilon}\left(2 \dot{H}-H^{2}\right)+H \ddot{\varepsilon}\right)-2 \dot{H}}{\kappa^{2} m^{4}}
\end{aligned}
$$

On the other hand, one can select the scalar field potential and the function $\varepsilon$, following string-inspired considerations:

$$
V=\Lambda+V_{0} e^{b_{1} \varphi}, \quad \varepsilon=\varepsilon_{0} e^{b_{2} \varphi} .
$$

Here $b_{1}$ and $b_{2}$ are constants. By analogy, the Lagrange multiplier is chosen in the same form

$$
\lambda=\lambda_{1}+\lambda_{0} e^{b_{3} \varphi},
$$

with $b_{3}$ being some constant.

Let us consider now some explicit cases to achieve solutions for the above dynamical system. It is clear that this is a sort of "inverse scattering procedure", that is by fixing the form of the scalar field, we solve dynamics. Usually, it is exactly the contrary: by fixing scalar field potentials and couplings, field equations gives the scalar field form.

\section{A. The case $\varphi \sim t$}

This choice of scalar $\varphi$ corresponds to a constant $U(\varphi)$. In addition, we assume that $\omega= \pm 1$. Hence,

$$
\varphi=\varphi_{0} t, \omega(\varphi)=\gamma= \pm 1
$$

where $\varphi_{0}$ is some constant.

One can solve the first Friedmann equation for the Hubble rate. In this case, the second equation becomes constant. We get:

$$
\begin{aligned}
H & =-\frac{e^{-\varphi_{0} b_{2} t}}{24 \varphi_{0} \varepsilon_{0} b_{2} \kappa^{2}}\left(\frac{1}{(1-2 \alpha+\sqrt{4 \alpha(\alpha-1)})^{1 / 3}}+\right. \\
& \left.+(1-2 \alpha+\sqrt{4 \alpha(\alpha-1)})^{1 / 3}+1\right)
\end{aligned}
$$

here $\alpha=72 \varphi_{0}^{2} \varepsilon_{0}^{2} b_{2}^{2} \kappa^{6} e^{2 \varphi_{0} b_{2} t}\left(\varphi_{0}^{2}(\gamma+2 \lambda)+2 V\right)$.

It is easy to see that $\alpha$ must be either less than zero or greater than one. That is $\varphi_{0}^{2}(\gamma+2 \lambda)+2 V<0$ or $\varphi_{0}^{2}(\gamma+2 \lambda)+2 V>1$.

Choosing the constant case, one can easily meet these requirements. If we assume that $\alpha$ is much bigger than one, then

$$
H \sim e^{-b_{2} \varphi} \sim 1 / \varepsilon
$$

For this case, it is easy to solve the Friedmann equation. Let the Hubble parameter is given by

$$
H=h_{0} e^{\varphi_{0} h t} .
$$

This is the so-called Little Rip cosmology [25] which can be realized also in the modified gravity context (see Refs. 27]). The effective EoS parameter of this model has the phantom nature, that means

$$
w_{e f f}=-1-\frac{2 \varphi_{0} h}{3 h_{0}} e^{-\varphi_{0} h t}
$$

In the Little Rip cosmology, $w$ approaches -1 sufficiently rapidly, so that it is possible to have a model in which $\rho_{D E}$ increases with time, but in which there is no finite-time future singularity. The characteristic feature of such a cosmology is disintegration of bound objects at finite time (like in the case of Big Rip cosmology).

As the universe expands, the relative acceleration between two points separated by a comoving distance $l$ is given by $l \ddot{a} / a$, where $a$ is the scale factor. An observer sitting at comoving distance $l$ away from a mass $m$ will measure an inertial force on the mass of the order

$$
F_{\text {iner }}=m l \ddot{a} / a=m l\left(\dot{H}+H^{2}\right) \text {. }
$$

Let us assume the two point force is bounded by the bounding force $F_{0}$. If $F_{\text {iner }}$ is positive and greater than $F_{0}$, the two particles become unbound. This effect is the "Rip" produced by the accelerating expansion. 
Eq. (19) shows that Rip occurs when either $H$ diverges or $\dot{H}$ diverges (assuming $\dot{H}>0$ ) [25, 26]. As we see, it is possible for $H$ and for $F_{\text {iner }}$, to increase without bound and yet not produce a future singularity at a finite time. This is the essence of Little Rip.

From Eq. (19) for (17), we have

$$
F_{\text {iner }}=m l\left(h_{0} \varphi_{0} h \mathrm{e}^{\varphi_{0} h t}+h_{0}^{2} \mathrm{e}^{2 \varphi_{0} h t}\right),
$$

which is positive and unbounded. Thus, $F_{\text {iner }}$ becomes arbitrary large with increasing $t$, that results in a Little Rip. The parameter $\varphi_{0} h$ can be estimated [25]. Let us assume

$$
\varphi_{0} h=\frac{\sqrt{3} \beta}{2}, \text { where } \beta \sim 3.46 \times 10^{-3} G_{y r^{-1}} .
$$

In addition, the present value of the Hubble constant is $H_{0}^{-1}=13.6$ Gyr $^{-1}$. In this case, one can estimate the time required for the disintegration of an object of the size of the Solar System as $t \sim 7750$ Gyr. Furthermore, it is easy to get the theory constants values $\varphi_{0} h=2.99 \times$ $10^{-3}, h_{0}=70.59 \times 10^{-3}$. For such a set of constants, the EoS parameter is equal to -1.0272 and this value does not contradict the observational bounds.

Let us make some general comments about the impact of the Lagrange multipliers on $F_{\text {iner }}$. From (19) and (7) one finds

$$
\begin{aligned}
& H^{2}+\dot{H}= \\
& \kappa^{2}\left(-\frac{2}{3} \gamma U-\frac{1}{3} \lambda U+\frac{1}{3} V+4 H^{3} \dot{\varepsilon}+8 H \dot{\varepsilon} \dot{H}+4 H^{2} \ddot{\varepsilon}\right) .
\end{aligned}
$$

It can be seen that, depending on the sign, $\lambda$ can either enhance or decrease $F_{\text {iner }}$. In other words, for realistic models, the role of Lagrange multiplier may be related to the increase/decrease of the time left before the disintegration of bound objects in the universe.

Let us now find the solution of the Friedmann equations (78) for the model (17) asuming the exponential scalar functions (12]13). There are two solutions:

$$
\begin{array}{rlrl}
V_{1} & =\frac{1}{2} \varphi_{0}^{2} \gamma-\frac{H^{2}}{\kappa^{2}} \quad \text { or } & V_{2}=\frac{1}{2} \varphi_{0}^{2} \gamma+3 \frac{H^{2}}{\kappa^{2}}, \\
\varepsilon_{1}=-\frac{1}{4 \varphi_{0} h h_{0} \kappa^{2}} \frac{1}{H} \quad \text { or } & \varepsilon_{2}=-\frac{1}{16 \kappa^{2}} \frac{1}{H^{2}}, \\
\lambda_{1}=-\gamma-\frac{2}{\varphi_{0}^{2} \kappa^{2}} H^{2} & \text { or } & \lambda_{2} & =-\gamma-\frac{3 h}{\varphi_{0} \kappa^{2}} H .
\end{array}
$$

In the first case, $\alpha$ tends to a constant value, which corresponds to the solution (15). In the second case $\alpha \rightarrow$ 0 .

Now we consider the case when $\varepsilon=0$. This solution is studied in Ref. [15]. Selecting the potential and Lagrange multiplier as (12) and (13), we do not get the solution in the form $H \sim e^{\varphi}$. However, it is easy to choose the potential in a slightly different form to get again the exponential Hubble rate.
Indeed, we have

$$
\begin{aligned}
H & =h_{0} e^{\varphi_{0} h t} \\
V & =\frac{1}{2} \varphi_{0}^{2} \gamma+\frac{2 \varphi_{0} h h_{0}}{\kappa^{2}} e^{\varphi_{0} h t}+\frac{3 h_{0}^{2}}{\kappa^{2}} e^{2 \varphi_{0} h t}, \\
\varepsilon & =0, \\
\lambda & =-\gamma-\frac{2 h_{0} h}{\varphi_{0} \kappa^{2}} e^{\varphi_{0} h t} .
\end{aligned}
$$

Once again, the Friedmann equations are satisfied. Obviously, this scheme may be applied to generate new cosmological solutions. It is clear that the potential and the Lagrange multiplier have now the form

$$
\begin{aligned}
V & =\frac{1}{\kappa^{2}}\left\{2 \dot{H}(t)+3 H(t)^{2}\right\}+\frac{\varphi_{0}^{2} \gamma}{2}, \\
\lambda & =-1-\frac{2}{\kappa^{2}} \dot{H}(t) .
\end{aligned}
$$

This solution has been obtained in ref.[13] in the same way.

As an example one can keep just a Lagrange multiplier $(V=0$ and $\varepsilon=0)$ in the form (13). Then solving the Friedmann equations, it is easy to find the Hubble rate

$$
H=-\frac{\varphi_{0} \sqrt{\gamma} \kappa}{\sqrt{6}} \tan \left[\frac{1}{2} \sqrt{\frac{3}{2}} \varphi_{0} \sqrt{\gamma} \kappa(t-\text { const })\right] .
$$

Since the solution is derived from the second Friedmann Eq. (8), there is no dependence on the Lagrange multiplier. Now using a Lagrangian multiplier, one can convert the first Friedmann Eq. (7) into an identity. In this case, we obtain $\lambda$ as follows

$$
\lambda=\frac{1}{2} \gamma\left(-1+\tan \left[\frac{1}{2} \sqrt{\frac{3}{2}} \varphi_{0} \sqrt{\gamma} \kappa(t-\text { const })\right]^{2}\right) .
$$

For this solution, EoS parameter $w$ lies in the range of $-1 / 3$ to infinity. In other words, the expansion is decelerating.

There is another solution, if $\gamma=-1$ (in this case, $H=$ const). The presence of the Lagrange multiplier leads to new solutions which do not exist without it. For example, using the conditions (12), (14) and (17) one cannot get a solution without a Lagrangian multiplier. If Lagrange multiplier (13) is taken into account then it is easy to construct the solution (23) and (24). This means that the presence of the Lagrange multiplier effectively changes the dynamical system giving rise to a constrained dynamics with, in principle, different solutions.

\section{B. The case $\varphi \sim \ln t$}

This form of scalar field allows us to choose the Hubble rate as $1 / t$ for the functions $V, \varepsilon$ and $\lambda$ given by Eqs. (12) and (13). 
That is

$$
H=\frac{h_{0}}{t}, \quad \varphi=\varphi_{0} \ln \frac{t}{t_{1}}, \omega(\varphi)=\gamma= \pm 1,
$$

where $h_{0}, \varphi_{0}$ and $t_{1}$ are some constants. When $h_{0}>0$, we have a quintessential power-law expansion. For

$$
H=-\frac{h_{0}}{t_{s}-t}, \quad \varphi=\varphi_{0} \ln \frac{t_{s}-t}{t_{1}}, \quad \omega(\varphi)=\gamma= \pm 1,
$$

we have a phantom power-law expansion when $h_{0}<0$. A phantom model describes the Big Rip finite-time future singularity: for $t=t_{s}$ the scale factor tends to infinity.

Now we can write the Friedmann equations as

$$
\begin{aligned}
& -\Lambda-\frac{\varphi_{0}^{2} \gamma}{2 t^{2}}+\frac{3 h_{0}^{2}}{k^{2} t^{2}}-\frac{\varphi_{0}^{2} \lambda_{1}}{t^{2}}-24 \varepsilon_{0} b_{2} h_{0}^{3} t^{-4+b_{2}} t_{1}^{-b_{2}}- \\
& -\varphi_{0}^{2} \lambda_{0} t^{-2+b_{3}} t_{1}^{-b_{3}}-t^{b_{1}} t_{1}^{-b_{1}} V_{0}=0 \\
& -\Lambda+\frac{\varphi_{0}^{2} \gamma}{2 t^{2}}-\frac{2 h_{0}}{k^{2} t^{2}}+\frac{3 h_{0}^{2}}{k^{2} t^{2}}+ \\
& +8 \varepsilon_{0} b_{2} h_{0}^{2}\left(3-b_{2}-2 h_{0}\right) t^{-4+b_{2}} t_{1}^{-b_{2}}-t^{b_{1}} t_{1}^{-b_{1}} V_{0}=0
\end{aligned}
$$

For $h_{0}>0$ (for $h_{0}<0$ we have to replace $t$ with $\left.t_{s}-t\right)$ and

$$
\begin{aligned}
V & =\Lambda+V_{0} e^{b_{1} \varphi / \varphi_{0}}, \quad \varepsilon=\varepsilon_{0} e^{b_{2} \varphi / \varphi_{0}} \\
\lambda & =\lambda_{1}+\lambda_{0} e^{b_{3} \varphi / \varphi_{0}}
\end{aligned}
$$

First, we consider the case when the Lagrangian multiplier and the potential is zero $(\lambda=V=0)$. It is easy to see, that

$$
\begin{aligned}
& b_{2}=2, \quad \varphi_{0}^{2}=-\frac{6 h_{0}^{2}\left(1-h_{0}\right)}{\gamma \kappa^{2}\left(1-5 h_{0}\right)} \\
& \varepsilon=\frac{\left(3 h_{0}-1\right)}{8 h_{0}\left(5 h_{0}-1\right) \kappa^{2}}(t s-t)^{2}, h_{0}<0
\end{aligned}
$$

or

$$
\varepsilon=\frac{\left(3 h_{0}-1\right)}{8 h_{0}\left(5 h_{0}-1\right) \kappa^{2}}(t)^{2}, h_{0}>0 \text {. }
$$

The solutions exist if

a) $\gamma<0$ and $h_{0}<0$ or $0<h_{0}<1 / 5\left(\right.$ or $h_{0}>1$ ),

b) $\gamma>0$ and $1 / 5<h_{0}<1$.

In the case in which the scalar field $\varphi$ is canonical $(\gamma=1)$, and there is no potential $(V(\varphi)=0)$, we cannot obtain the effective phantom cosmological solution with $w<-1$. A similar situation is obtained for $\varepsilon=0$ and $V \neq 0$. In this case, the potential has the form

$$
\begin{aligned}
V & =\frac{h_{0}\left(3 h_{0}-1\right)}{\kappa^{2} t^{2}}, \text { for } h_{0}>0 \\
\text { or } V & =\frac{h_{0}\left(3 h_{0}-1\right)}{\kappa^{2}\left(t_{s}-t\right)^{2}}, \text { for } h_{0}<0
\end{aligned}
$$

In addition there are restrictions on $h_{0}: \varphi_{0}^{2} \gamma=2 h_{0} / \kappa^{2}$. One sees that $w<-1$ only in the case $\gamma<0$.
Now let $\lambda=0$ but $V \neq 0$ and $\varepsilon \neq 0$. In this case, the solution exists only for $b_{2}=-b_{1}=2$ [13]. Then

$$
\begin{aligned}
\varepsilon= & \frac{\left(2 h_{0}-\varphi_{0}^{2} \gamma k^{2}\right) t^{2}}{16 h_{0}^{2}\left(1+h_{0}\right) k^{2}} \\
V= & \frac{6\left(-1+h_{0}\right) h_{0}^{2}+\varphi_{0}^{2} \gamma\left(-1+5 h_{0}\right) k^{2}}{2\left(1+h_{0}\right) k^{2} t^{2}} \\
& h_{0}>0 \text { or } t \rightarrow t_{s}-t, \text { if } h_{0}<0 .
\end{aligned}
$$

It is evident that there is a phantom solution for $\gamma>0$.

If $\lambda \neq 0$ and $V=0$, we obtain the following conditions restricting $h_{0}$

$$
\varphi_{0}^{2}=-\frac{2 h_{0}^{2}\left(3 h_{0}-2\right)}{\gamma \kappa^{2}}
$$

Therefore, to get the value of $w$ about $-1, \gamma$ must be negative. If $\gamma>0$, then $h_{0}$ has to lie in the following range $0<h_{0}<2 / 3$. For this case, we have

$$
\begin{aligned}
\varepsilon= & \varepsilon_{0}\left(\frac{t}{t_{1}}\right)^{3-2 h_{0}}, \\
\lambda= & \frac{\gamma\left(1-3 h_{0}\right)}{3 h_{0}-2}-\frac{12 \varepsilon_{0} \gamma h_{0}^{2}\left(2 h_{0}-3\right)}{\left(3 h_{0}-2\right) t_{1}^{2}}\left(\frac{t}{t_{1}}\right)^{1-2 h_{0}} \\
& \quad \text { for } h_{0}>0 \text { and } t \rightarrow t_{s}-t \text { if } h_{0}<0 .
\end{aligned}
$$

For sufficiently large $h_{0}$, the function $\varepsilon$ has a negative power and quickly decreases with time (for $h_{0}>0$ ) or increases when approaching to $t_{s}$ (for $h_{0}<0$ ). For the model without a Lagrangian multiplier (33), function $\varepsilon$ grows for $h_{0}>0$ and decreases for $h_{0}<0$.

If $\lambda \neq 0$ and $\varepsilon=0$, we get

$$
\lambda=-\gamma+\frac{2 h_{0}}{\varphi_{0}^{2} \kappa^{2}}, \quad V=\frac{-4 h_{0}+6 h_{0}^{2}+\varphi_{0}^{2} \gamma \kappa^{2}}{2 \kappa^{2} t^{2}} .
$$

Such behavior is similar to the one in the model without the Lagrange multiplier (33).

Suppose now that the action has the form (2), that is $V \neq 0, \varepsilon \neq 0$ and $\lambda \neq 0$. From Eqs.(30), one sees that there are several solutions:

1) $b_{1}=-4+b_{2}$. For this case, there are two additional conditions: $b_{2}=2$ and $b_{2} \neq 2$.

In the first case it is easy to find:

$$
\begin{aligned}
V & =\frac{32 \varepsilon_{0}\left(1-2 h_{0}\right) h_{0}^{2} \kappa^{2}+\left(2 h_{0}\left(3 h_{0}-2\right)+\varphi_{0}^{2} \gamma \kappa^{2}\right) t_{1}^{2}}{2 \kappa^{2} t_{1}^{2} t^{2}} \\
\varepsilon & =\varepsilon_{0} \frac{t^{2}}{t_{1}^{2}}, \\
\lambda & =\frac{-16 \varepsilon_{0} h_{0}^{3}\left(1+h_{0}\right) \kappa^{2}+\left(2 h_{0}-\varphi_{0}^{2} \gamma \kappa^{2}\right) t_{1}^{2}}{\varphi_{0}^{2} \kappa^{2} t_{1}^{2}}=\text { const. }
\end{aligned}
$$

This means that the solution is similar to the solution for the case without Lagrange multiplier (35). The time dependence is the same. The difference enters via an arbitrary factor $\varepsilon_{0}$ by choosing the Lagrange multiplier. 
Now, let $b_{2} \neq 2$, then we obtain the following condition

$$
\varphi_{0}= \pm \frac{\sqrt{2}}{\kappa} \sqrt{\frac{h_{0}\left(2-3 h_{0}\right)}{\gamma}} .
$$

Again limits appear on the Hubble rate. Phantom cosmology is possible only for $\gamma<0$. If $\gamma>0$, then $h_{0}$ must be greater than zero and less than $2 / 3$. For this case, we have

$$
\begin{aligned}
V & =-\frac{8 \varepsilon_{0} b_{2} h_{0}\left(b_{2}-3+2 h_{0}\right)\left(t / t_{1}\right)^{-4+b_{2}}}{t_{1}^{4}}, \\
\varepsilon & =\varepsilon_{0} \frac{t^{b_{2}}}{t_{1}^{b_{2}}}, \\
\lambda & =\gamma \frac{1-3 h_{0}}{3 h_{0}-2}+\frac{4 \varepsilon_{0} b_{2} \gamma\left(b_{2}-3-h_{0}\right) h_{0} \kappa^{2}}{\left(2-3 h_{0}\right) t_{1}^{2}}\left(\frac{t}{t_{1}}\right)^{-2+b_{2}} .
\end{aligned}
$$

2) Assume now the following conditions $b_{2}=3-2 h_{0}$. Then

$$
\begin{aligned}
V & =\frac{-4 h_{0}+6 h_{0}^{2}+\varphi_{0}^{2} \gamma \kappa^{2}}{2 \kappa^{2} t^{2}}, \\
\varepsilon & =\varepsilon_{0}\left(\frac{t}{t_{1}}\right)^{3-2 h_{0}}, \\
\lambda & =-\gamma+\frac{2 h_{0}}{\varphi_{0}^{2} k^{2}}+\frac{24 \varepsilon_{0} h_{0}^{3}\left(-3+2 h_{0}\right)\left(\frac{t}{t_{1}}\right)^{1-2 h_{0}}}{\varphi_{0}^{2} t_{1}^{2}} .
\end{aligned}
$$

3) There is one more case: $b_{2}=4$. Then

$$
\begin{aligned}
V & =-\frac{32 \varepsilon_{0} h_{0}^{2}\left(1+2 h_{0}\right)}{t_{1}^{4}}+\frac{-4 h_{0}+6 h_{0}^{2}+\varphi_{0}^{2} \gamma \kappa^{2}}{2 \kappa^{2} t^{2}}, \\
\varepsilon & =\varepsilon_{0}\left(\frac{t}{t_{1}}\right)^{4} \\
\lambda & =-\gamma+\frac{2 h_{0}}{\varphi_{0}^{2} k^{2}}+\frac{32 \varepsilon_{0} h_{0}^{2}\left(1-h_{0}\right) t^{2}}{\varphi_{0}^{2} t_{1}^{4}} .
\end{aligned}
$$

Hence, the Lagrange multiplier presence helps to generate new cosmological solutions. For models without such multipliers, we find that the potential is proportional to the square of the time, while the scalar function of the Gauss-Bonnet invariant is inversely proportional to the time. In the model with the Lagrange multiplier we get new solutions (37), (40), (41) and (42). In all these cases, the dependence of the coefficient of the Gauss-Bonnet invariant is explicit and simple, whereas the scalar potential often shows the same time dependence $\left(V \sim 1 / t^{2}\right)$. It should also be noted that there may occur phantom cosmologies for the canonical scalar field $\varphi$. Only for solutions (40) we obtain restrictions on $h_{0}$ (for $V, \varepsilon$ and $\lambda \neq 0$ ).

Without the Lagrange multiplier, the scalar potential increases with time as $t^{2}$ (if $h_{0}>0$ ), and the function $\varepsilon$ decreases as $1 / t^{2}$, or vice-versa if $h_{0}<0$. In presence of the Lagrange multiplier, one can get exactly the same behavior as well as new solutions: $\varepsilon$ and $V$ increase (for $\left.h_{0}>0\right)$ or another case where the potential decreases as $1 / t^{2}$, and $\varepsilon$ increases as $t^{3-2 h_{0}}$ (for $h_{0}<0$ ) or decreases (for $h_{0}<0$ ). Let us consider the case with/without the Lagrange multiplier but with the same scalar potentials. Let $b_{1}=-2, \quad b_{2}=2$ and $h_{0}=-80 / 3, \kappa=1, \gamma=1$. In this case, we numerically derive

$$
\begin{aligned}
V_{0} & =2304.8+4.23377 \lambda \\
\varepsilon & =0.000189478+6.84862 * 10^{-6} \lambda .
\end{aligned}
$$

The Big Rip cosmology occurs with the corresponding Rip time for model (29) at $t_{s}=376.27$ Gyr. In summary, the presence of Lagrange multiplier may lead to the generation of new cosmological solutions if compared with scalar Gauss-Bonnet gravity without such term. The general reason for this different behavior will be discussed below.

\section{THE RECONSTRUCTION OF SCALAR-GAUSS-BONNET GRAVITY WITH LAGRANGE MULTIPLIER}

In this section we discuss the general scheme of cosmological reconstruction in the scalar-Gauss-Bonnet gravity with the Lagrange multiplier. Let $\omega(\varphi)$ be 1. Friedmann Eqs. (7-8) can be written as

$$
\begin{aligned}
& \frac{3}{\kappa^{2}} H^{2}= \\
& =(1+2 \lambda(\varphi)) \frac{\dot{\varphi}^{2}}{2}+V(\varphi)+24 H^{3} \frac{d \varepsilon(\varphi(t))}{d t}, \\
& \frac{-1}{\kappa^{2}}\left(2 \dot{H}+3 H^{2}\right)=\frac{\dot{\varphi}^{2}}{2}-V(\varphi)-8 H^{2} \frac{d^{2} \varepsilon(\varphi(t))}{d t^{2}}- \\
& -16 H \dot{H} \frac{d \varepsilon(\varphi(t))}{d t}-16 H^{3} \frac{d \varepsilon(\varphi(t))}{d t} .
\end{aligned}
$$

It is not difficult to express the Lagrange multiplier and the potential in terms of the other functions. In this way we get them by fixing the metric, the scalar field and the coefficient of the Gauss-Bonnet invariant. They are

$$
\begin{aligned}
& \lambda= \\
& =\dot{\varphi}^{-2}\left(-\frac{2}{\kappa^{2}} \dot{H}-\frac{\dot{\varphi}^{2}}{2}-8 H^{2} \frac{d^{2} \varepsilon}{d t^{2}}-8 H \frac{d \varepsilon}{d t}\left(\dot{H}-H^{2}\right)\right), \\
& V= \\
& =\frac{2 \dot{H}+3 H^{2}}{\kappa^{2}}+\frac{\dot{\varphi}^{2}}{2}-8 H^{2} \frac{d^{2} \varepsilon}{d t^{2}}-16 H \frac{d \varepsilon}{d t}\left(\dot{H}+H^{2}\right) .
\end{aligned}
$$

Using these expressions, it is easy to verify the validity of earlier solutions.

Let us consider now the case when, as unknown functions do not appears the Lagrange multiplier and the function $\varepsilon(\varphi)$. Combining the Friedmann Eqs. (44) and 
(45), we obtain

$$
\begin{aligned}
0 & =\frac{2}{\kappa^{2}} \dot{H}+\dot{\varphi}^{2}+\lambda(\varphi) \dot{\varphi}^{2}-8 H^{2} \frac{d^{2} \varepsilon(\varphi(t))}{d t^{2}}- \\
& -16 H \dot{H} \frac{d \varepsilon(\varphi(t))}{d t}+8 H^{3} \frac{d \varepsilon(\varphi(t))}{d t} \\
& =\frac{2}{\kappa^{2}} \dot{H}+\dot{\varphi}^{2}+\lambda(\varphi) \dot{\varphi}^{2}-8 a \frac{d}{d t}\left(\frac{H^{2}}{a} \frac{d \varepsilon(\varphi(t))}{d t}\right) .
\end{aligned}
$$

Eq.(48) can be solved with respect to $\varepsilon(\varphi(t))$ as

$$
\begin{aligned}
& \varepsilon(\varphi(t))=\frac{1}{8} \int^{t} d t_{1} \frac{a\left(t_{1}\right)}{H\left(t_{1}\right)^{2}} \times \\
& \times \int^{t_{1}} \frac{d t_{2}}{a\left(t_{2}\right)}\left(\frac{2}{\kappa^{2}} \dot{H}\left(t_{2}\right)+\dot{\varphi}\left(t_{2}\right)^{2}+\lambda\left(\varphi\left(t_{2}\right)\right) \dot{\varphi}\left(t_{2}\right)^{2}\right) .
\end{aligned}
$$

Combining Eqs. (44) and (49), the scalar potential $V(\varphi(t))$ is:

$$
\begin{aligned}
& V(\varphi(t))=\frac{3}{\kappa^{2}} H(t)^{2}-\frac{1}{2} \dot{\varphi}(t)^{2}-\lambda(\varphi(t)) \dot{\varphi}(t)^{2}- \\
& -3 a(t) H(t) \int^{t} \frac{d t_{1}}{a\left(t_{1}\right)} \times \\
& \times\left(\frac{2}{\kappa^{2}} \dot{H}\left(t_{1}\right)+\dot{\varphi}\left(t_{1}\right)^{2}+\lambda\left(\varphi\left(t_{1}\right)\right) \dot{\varphi}\left(t_{1}\right)^{2}\right) .
\end{aligned}
$$

Let us identify $t$ with $f(\varphi)$ and $H$ with $g^{\prime}(t)$ where $f$ and $g$ are some unknown functions. The solution of the Friedmann equations is related to the existence and behavior of such functions. Then we consider a model where $V(\varphi)$ and $\varepsilon(\varphi)$ can be expressed in terms of the two functions $f$ and $g$ as

$$
\begin{aligned}
V(\varphi) & =\frac{3}{\kappa^{2}} g^{\prime}(f(\varphi))^{2}-\frac{1}{2 f^{\prime}(\varphi)^{2}}-\frac{\lambda(\varphi)}{f^{\prime}(\varphi)^{2}}- \\
& -3 g^{\prime}(f(\varphi)) \mathrm{e}^{g(f(\varphi))} \int^{\varphi} d \varphi_{1} f^{\prime}\left(\varphi_{1}\right) \mathrm{e}^{-g\left(f\left(\varphi_{1}\right)\right)} \times \\
& \times\left(\frac{2}{\kappa^{2}} g^{\prime \prime}\left(f\left(\varphi_{1}\right)\right)+\frac{1}{f^{\prime}\left(\varphi_{1}\right)^{2}}+\frac{\lambda\left(\varphi_{1}\right)}{f^{\prime}\left(\varphi_{1}\right)^{2}}\right)= \\
& =\frac{3}{\kappa^{2}} g^{\prime}(f(\varphi))^{2}-\frac{1}{2 f^{\prime}(\varphi)^{2}}- \\
& -3 g^{\prime}(f(\varphi)) \mathrm{e}^{g(f(\varphi))} \int^{\varphi} d \varphi_{1} f^{\prime}\left(\varphi_{1}\right) \mathrm{e}^{-g\left(f\left(\varphi_{1}\right)\right)} \times \\
& \times\left(\frac{2}{\kappa^{2}} g^{\prime \prime}\left(f\left(\varphi_{1}\right)\right)+\frac{1}{f^{\prime}\left(\varphi_{1}\right)^{2}}\right)- \\
& -\frac{\lambda(\varphi)}{f^{\prime}(\varphi)^{2}}-3 g^{\prime}(f(\varphi)) \mathrm{e}^{g(f(\varphi))} \times \\
& \times \int^{\varphi} d \varphi_{1} \mathrm{e}^{-g\left(f\left(\varphi_{1}\right)\right)}\left(\frac{\lambda\left(\varphi_{1}\right)}{f^{\prime}\left(\varphi_{1}\right)}\right)
\end{aligned}
$$

$$
\begin{aligned}
\varepsilon(\varphi) & =\frac{1}{8} \int^{\varphi} d \varphi_{1} \frac{f^{\prime}\left(\varphi_{1}\right) \mathrm{e}^{g\left(f\left(\varphi_{1}\right)\right)}}{g^{\prime}\left(\varphi_{1}\right)^{2}} \times \\
& \times \int^{\varphi_{1}} d \varphi_{2} f^{\prime}\left(\varphi_{2}\right) \mathrm{e}^{-g\left(f\left(\varphi_{2}\right)\right)} \times \\
& \times\left(\frac{2}{\kappa^{2}} g^{\prime \prime}\left(f\left(\varphi_{2}\right)\right)+\frac{1}{f^{\prime}\left(\varphi_{2}\right)^{2}}+\frac{\lambda\left(\varphi_{2}\right)}{f^{\prime}\left(\varphi_{2}\right)^{2}}\right)= \\
& =\frac{1}{8} \int^{\varphi} d \varphi_{1} \frac{f^{\prime}\left(\varphi_{1}\right) \mathrm{e}^{g\left(f\left(\varphi_{1}\right)\right)}}{g^{\prime}\left(\varphi_{1}\right)^{2}} \times \\
& \times \int^{\varphi_{1}} d \varphi_{2} f^{\prime}\left(\varphi_{2}\right) \mathrm{e}^{-g\left(f\left(\varphi_{2}\right)\right)} \times \\
& \times\left(\frac{2}{\kappa^{2}} g^{\prime \prime}\left(f\left(\varphi_{2}\right)\right)+\frac{1}{f^{\prime}\left(\varphi_{2}\right)^{2}}\right)+ \\
& +\frac{1}{8} \int^{\varphi} d \varphi_{1} \frac{f^{\prime}\left(\varphi_{1}\right) \mathrm{e}^{g\left(f\left(\varphi_{1}\right)\right)}}{g^{\prime}\left(\varphi_{1}\right)^{2}} \times \\
& \times \int^{\varphi_{1}} d \varphi_{2} \mathrm{e}^{-g\left(f\left(\varphi_{2}\right)\right)}\left(\frac{\lambda\left(\varphi_{2}\right)}{f^{\prime}\left(\varphi_{2}\right)}\right) .
\end{aligned}
$$

By choosing $V(\varphi)$ and $\varepsilon(\varphi)$ as in Eqs. (51), one can easily find the following solution for Eqs.(44) and (45) that can be compared with results in [15]), that is

$$
\begin{gathered}
\varphi=f^{-1}(t) \quad(t=f(\varphi)), \\
a=a_{0} \mathrm{e}^{g(t)} \quad\left(H=g^{\prime}(t)\right) .
\end{gathered}
$$

Hence any monotonic evolving cosmology, expressed by $H=g^{\prime}(\varphi)$ in the model (2) with the potential (51), can be realized, including models exhibiting the transition from non-phantom phase to phantom phase without introducing the phantom scalar field. However, we have to note that the approach could become problematic for $H(t)$ non-monotonic as we will discuss in the next subsection.

As an example, let us consider the model (28). Hence,

$$
\begin{aligned}
& f(\varphi)=t=t_{1} e^{\varphi / \varphi_{0}}, \quad f^{\prime}(\varphi)=\frac{t_{1}}{\varphi_{0}} e^{\varphi / \varphi_{0}}, \\
& g(\varphi)=h_{0} \frac{\varphi_{0}}{\varphi}, \quad g^{\prime}(\varphi)=\frac{h_{0}}{t_{1}} e^{-\varphi / \varphi_{0}},
\end{aligned}
$$

where prime is the time derivative $\left({ }^{\prime}=d / d t\right)$. Now we choose the Lagrange multiplier as in Eqs. (42). In this case, the potential and the function $\varepsilon$ iare composed by two terms: the first term is the value in the absence of a Lagrange multiplier. For $\varepsilon$, it has the form

$$
\frac{t_{1}^{2}\left(2 h_{0}-\varphi_{0}^{2} \kappa^{2}\right)}{16 h_{0}^{2}\left(1+h_{0}^{2}\right) \kappa^{2}} e^{2 \varphi / \varphi_{0}} .
$$

If $e^{2 \varphi / \varphi_{0}}=t^{2} / t_{1}^{2}$, this expression is equal to (35). The second term (52) has the form

$$
e^{2 \varphi / \varphi_{0}} \frac{\left(16 e^{2 \varphi / \varphi_{0}} \varepsilon_{0} h_{0}^{2}\left(1+h_{0}\right) \kappa^{2}+\left(\varphi_{0}^{2} \kappa^{2}-2 h_{0}\right) t_{1}^{2}\right)}{16 h_{0}^{2}\left(1+h_{0}\right) \kappa^{2}} .
$$

It also contains the first term with a different sign, and also the following expression

$$
\varepsilon_{0} e^{4 \varphi / \varphi_{0}}=\varepsilon_{0}\left(\frac{t}{t_{1}}\right)^{4},
$$


which coincides with Eq.(42). This example is paradigmatic to show how the reconstruction scheme works for the Gauss-Bonnet gravity with Lagrange multiplier.

\section{A. The case of non-monotonic functions of time.}

The above examples correspond to monotonic behaviors in time for the Hubble parameter and the scalar field. However, from Eqs. (51) and (52), one could run into singularities emerging from non-monotonic behaviors (for example $\dot{H}=0$ and $\dot{\varphi}=0$ for some values of $t$ ). If we consider the original Eqs. (44) and (45), we see that terms like $1 / \dot{H}$ and $1 / \dot{\varphi}$ do not appear. The problem may occur when we recast $V$ and $\varepsilon$ in terms of $\varphi$. To face the issue of non-monotonic behaviors and construct suitable examples, let us take into account the following form of the Hubble parameter

$$
H=h_{0}\left(\frac{1}{t}+\frac{1}{t_{0}-t}\right) .
$$

This means that the universe is in a non-phantom phase for $t<t_{0} / 2$ and in a phantom phase for $t>t_{0} / 2$. There is also a Big Rip singularity for $t=t_{0}$ and a point where one can define an effective cosmological constant for $t=t_{0} / 2$ $\left(\dot{H}\left(t_{0} / 2\right)=0\right)$.

One can take into account two different behaviors for the scalar field:

1) $\varphi=\varphi_{0} t$, that is $t=f(\varphi)=\varphi / \varphi_{0}$ and

2) $\varphi=\varphi_{0} \ln \left(t\left(t_{0}-t\right)\right)$, that is $t=f(\varphi)=$

$=\frac{1}{2}\left(t_{0} \pm \sqrt{t_{0}^{2}-4 e^{\varphi / \varphi_{0}}}\right)$. Clearly, expressing $V$ and $\varepsilon$ by $\varphi$, uncertainties on the time evolution can come out while they are removed as soon as time-dependence in considered.

Let us derive $V$ and $\varepsilon$ for the first case. The general case contains special functions under the integrals and then we consider the specific case $h_{0}=2$. We have

$$
\begin{aligned}
\varepsilon & =\frac{1}{32 \varphi_{0}^{2} \varphi_{s}^{2}}\left(\frac{\varphi^{6}}{6}+\frac{c_{1} \varphi^{5}}{5}+c_{2}+\frac{2 \varphi^{5} \varphi_{s}}{25}-\frac{\varphi^{4} \varphi_{s}^{2}}{4}+\right. \\
& \left.+\frac{8 \varphi^{5}}{15 \varphi_{s} \kappa^{2}}-\frac{4 \varphi^{3} \varphi_{s}}{3 \kappa^{2}}+\frac{2 \varphi^{2} \varphi_{s}^{2}}{3 \kappa^{2}}-\frac{2}{5} \varphi^{5} \varphi_{s} \ln \frac{\varphi}{\varphi_{s}}\right)+ \\
& +\frac{1}{8} \int^{\varphi} d \varphi_{1} \frac{f^{\prime}\left(\varphi_{1}\right) \mathrm{e}^{g\left(f\left(\varphi_{1}\right)\right)}}{g^{\prime}\left(\varphi_{1}\right)^{2}} \times \\
& \times \int^{\varphi_{1}} d \varphi_{2} \mathrm{e}^{-g\left(f\left(\varphi_{2}\right)\right)}\left(\frac{\lambda\left(\varphi_{2}\right)}{f^{\prime}\left(\varphi_{2}\right)}\right) \\
& =\frac{4 \varphi_{0}^{2}\left(2 \varphi+\varphi_{s}\right)^{2}}{\varphi^{2}\left(\varphi-\varphi_{s}\right)^{2} \kappa^{2}}-\frac{12 \varphi_{0}^{2} \varphi \varphi_{s}^{2} \ln \frac{\varphi}{\varphi_{s}}}{\left(\varphi^{2}-\varphi_{s}\right)^{3}}+ \\
& +\frac{2 c_{1}\left(\varphi-\varphi_{s}\right)^{2}+\varphi_{0}^{2}\left(-\varphi^{2}+14 \varphi_{s}+11 \varphi_{s}^{2}\right)}{2\left(\varphi-\varphi_{s}\right)^{2}}- \\
& -\frac{\lambda(\varphi)}{f^{\prime}(\varphi)^{2}}-3 g^{\prime}(f(\varphi)) \mathrm{e}^{g(f(\varphi))} \times \\
& \times \int^{\varphi} d \varphi_{1} \mathrm{e}^{-g\left(f\left(\varphi_{1}\right)\right)}\left(\frac{\lambda\left(\varphi_{1}\right)}{f^{\prime}\left(\varphi_{1}\right)}\right)
\end{aligned}
$$

where $\varphi_{s}=\varphi_{0} t_{0}$. We see that by choosing different types of functions $\lambda$, we obtain different forms of $V$ and $\varepsilon$. It is not always easy to find the resulting integral: for example, choosing $\lambda=\lambda_{0} e^{\varphi / \varphi_{0}}$, we obtain the solution in terms of the Euler function. However, it is easy to obtain solutions for the case $\lambda=\lambda_{0}\left(\varphi / \varphi_{0}\right)^{\lambda_{1}}$. We have

$$
\begin{aligned}
\varepsilon & =\frac{1}{32 \varphi_{0}^{2} \varphi_{s}^{2}}\left(\frac{\varphi^{6}}{6}+\frac{c_{1} \varphi^{5}}{5}+c_{2}+\frac{2 \varphi^{5} \varphi_{s}}{25}-\frac{\varphi^{4} \varphi_{s}^{2}}{4}+\right. \\
& +\frac{8 \varphi^{5}}{15 \varphi_{s} \kappa^{2}}-\frac{4 \varphi^{3} \varphi_{s}}{3 \kappa^{2}}+\frac{2 \varphi^{2} \varphi_{s}^{2}}{3 \kappa^{2}}-\frac{2}{5} \varphi^{5} \varphi_{s} \ln \frac{\varphi}{\varphi_{s}}+ \\
& +\frac{\varphi^{4}\left(\frac{\varphi}{\varphi_{0}}\right)^{\lambda_{1}} \lambda_{0}}{\left(\lambda_{1}-1\right) \lambda_{1}\left(1+\lambda_{1}\right)\left(4+\lambda_{1}\right)\left(5+\lambda_{1}\right)\left(6+\lambda_{1}\right)} \times \\
& \times\left(\varphi^{2}\left(\lambda_{1}-1\right) \lambda_{1}\left(4+\lambda_{1}\right)\left(5+\lambda_{1}\right)-\right. \\
& -2 \varphi_{s}\left(\lambda_{1}-1\right)\left(1+\lambda_{1}\right)\left(4+\lambda_{1}\right)\left(6+\lambda_{1}\right)+ \\
& \left.\left.+\varphi_{s}^{2} \lambda_{1}\left(1+\lambda_{1}\right)\left(5+\lambda_{1}\right)\left(6+\lambda_{1}\right)\right)\right)
\end{aligned}
$$

$$
\begin{aligned}
V & =\frac{4 \varphi_{0}^{2}\left(2 \varphi+\varphi_{s}\right)^{2}}{\varphi^{2}\left(\varphi-\varphi_{s}\right)^{2} \kappa^{2}}-\frac{12 \varphi_{0}^{2} \varphi \varphi_{s}^{2} \ln \frac{\varphi}{\varphi_{s}}}{\left(\varphi-\varphi_{s}\right)^{3}}+ \\
& +\frac{2 c_{1}\left(\varphi-\varphi_{s}\right)^{2}+\varphi_{0}^{2}\left(-\varphi^{2}+14 \varphi \varphi_{s}+11 \varphi_{s}^{2}\right)}{2\left(\varphi-\varphi_{s}\right)^{2}}- \\
& -\frac{\varphi_{0}^{2}\left(\frac{\varphi}{\varphi_{0}}\right)^{\lambda_{1}} \lambda_{0}}{\left(\varphi-\varphi_{s}\right)^{3} \lambda_{1}\left(\lambda_{1}^{2}-1\right)} \times \\
& \times\left(-3 \varphi^{2} \varphi_{s}\left(-1+\lambda_{1}\right) \lambda_{1}\left(3+\lambda_{1}\right)+\right. \\
& +3 \varphi_{s}^{2}\left(-1+\lambda_{1}\right)\left(1+\lambda_{1}\right)\left(4+\lambda_{1}\right)- \\
& \left.-\varphi_{s}^{3} \lambda_{1}\left(1+\lambda_{1}\right)\left(5+\lambda_{1}\right)+\varphi^{3} \lambda_{1}\left(-1+\lambda_{1}^{2}\right)\right)
\end{aligned}
$$

This means that the delicate point is related to a suitable choice of $\lambda$.

Let us consider now the second case where the scalar field is a logarithmic function of time. We assume again $h_{0}=2$ and $\lambda=\lambda_{0} e^{-2 \varphi / \varphi_{0}}$. For this choice, we obtain solutions that do not contain special functions, that is

$$
\begin{aligned}
\varepsilon & =\frac{\varphi_{0}^{2}}{80 t_{0}^{2}}\left(\frac{20 c_{1} t^{7}}{7 \varphi_{0}^{2}}-\frac{5}{4} \varphi_{0}^{2} \kappa^{2} t^{8}-\frac{2 t^{5} t_{0}}{\varphi_{0}^{2} \kappa^{2}}+\varphi_{0}^{2} \kappa^{2} t^{5} t_{0}+c_{2}+\right. \\
& +\frac{5 t^{4} t_{0}^{2}}{6 \varphi_{0}^{2} \kappa^{2}}-\frac{5}{24} \varphi_{0}^{2} \kappa^{2} t^{4} t_{0}^{4}--\frac{\lambda_{0} t^{4}}{t_{0}^{4}}-\frac{\lambda_{0} t^{3}}{2 t_{0}^{3}}-\frac{\lambda_{0} t^{2}}{3 t_{0}^{2}}- \\
& -\frac{\lambda_{0} t}{4 t_{0}}-\frac{1}{2} \lambda_{0} \ln [t]+\frac{\lambda_{0} t^{5} \ln [t]}{t_{0}^{5}}-\frac{3}{2} \lambda_{0} \ln \left[t_{0}-t\right]- \\
& \left.-\frac{\lambda_{0} t^{5} \ln \left[t_{0}-t\right]}{t_{0}^{5}}\right),
\end{aligned}
$$




$$
\begin{aligned}
V & =-\frac{\varphi_{0}^{2}\left(4 t^{3}+40 t^{2} t_{0}-19 t t_{0}^{2}+3 t_{0}^{3}\right)}{2 t^{2}\left(t-t_{0}\right)^{3}}- \\
& -\frac{4 t_{0}\left(-3 c_{1} \kappa^{2} t^{3}+2 t_{0}\left(3 t+t_{0}\right)\right)}{2 \kappa^{2} t^{2}\left(t-t_{0}\right)^{3}}+ \\
& +\frac{\varphi_{0}^{2} \lambda_{0}\left(-60 t^{5}+30 t^{4} t_{0}+10 t^{3} t_{0}^{2}-15 t^{2} t_{0}^{3}-t t_{0}^{4}+t_{0}^{5}\right)}{5 t^{4}\left(t-t_{0}\right)^{4} t_{0}^{3}}+ \\
& +\frac{60 \varphi_{0}^{2} \lambda_{0} t\left(\ln [t]-\ln \left[t_{0}-t\right]\right)}{5\left(t-t_{0}\right)^{3} t_{0}^{4}}
\end{aligned}
$$

In this case, we have written the expression as a function of time, as we have the uncertainty noted above.

In conclusion, it can be seen that the method works not only for monotonic functions, but also for some nonmonotonic Hubble functions that have to be carefully considered. However problems may arise by converting the functions $V$ and $\varepsilon$ in terms of the scalar field. In this process, uncertainty on the behavior can arise. The interest to derive suitable Hubble rate forms (not necessarily monotonic) is related to the necessity to obtain realistic dark energy models. In fact, $H(t)$ decreasing with time is generally related to the evolution of matter dominated epochs and large scale structure formation. On the other hand, $H(t)$ increasing with time can be related to inflationary and dark energy behaviors. Clearly, special care has to be devoted to singularities at finite which could come out, as above, in this reconstruction process.

\section{DYNAMICAL SYSTEM ANALYSIS AND CRITICAL POINTS}

Let us rewrite the cosmological Friedmann equations in terms of an autonomous dynamical system. The approach consists of two parts: the Friedmann Eqs. (7) and (8) and equations specifying the model, for example (12) and (13). It is convenient to work with the new variables

$$
x=\frac{\dot{\varphi}}{H}, \quad y=\dot{\varepsilon} H, \quad z=\frac{V}{H^{2}}, \quad \mu=\frac{\dot{H}}{H^{2}} .
$$

The prime means the derivative with respect to $\ln a$.

After some algebra, we obtain the following dynamical system

$$
\begin{aligned}
0 & =-\frac{3}{\kappa^{2}}+\frac{1}{2} x^{2}+\lambda x^{2}+z+24 y \\
0 & =\frac{1}{\kappa^{2}}(2 \mu+3)+\frac{1}{2} x^{2}-z-8 y^{\prime}-8 y \mu-16 y, \\
y^{\prime} & =y\left(b_{2} x+\frac{x^{\prime}}{x}+2 \mu\right) \\
z^{\prime} & =b_{1} z x-2 z \mu-\frac{b_{1} \Lambda x}{H^{2}} \\
\lambda^{\prime} & =b_{3}\left(\lambda-\lambda_{1}\right) x
\end{aligned}
$$

where the first equation is a constraint for the other 4 equations. For the sake of simplicity, we have considerd the case $\Lambda=0$. As usual, the critical points at finite are given by the condition

$$
x^{\prime}=y^{\prime}=z^{\prime}=\lambda^{\prime}=0 .
$$

The various situations are

- $\mathbf{A}:(x, y, z, \lambda)=\left(0,0, \frac{3}{\kappa^{2}}, \lambda\right)$ or $=\left(0,0, \frac{3}{\kappa^{2}}, \lambda_{1}\right)$ (two points - any $\lambda$ or $\lambda=\lambda_{1}$ is constant). For this point $\mu=0$ and hence EoS parameter is equal to minus one $(w=-1)$. Thus, it is a de Sitter space. In this point, the potential of the scalar field dominates.

- $\mathbf{B}:(x, y, z, \lambda)=\left(-\frac{b_{1}}{\kappa^{2}\left(1+\lambda_{1}\right)}, 0, \frac{6 \kappa^{2}\left(1+\lambda_{1}\right)-b_{1}\left(1+2 \lambda_{1}\right)}{2 \kappa^{4}\left(1+\lambda_{1}\right)^{2}}, \lambda_{1}\right)$ ( $\lambda$ is constant). For this point $\mu=-\frac{b_{1}^{2}}{2 \kappa^{2}\left(1+\lambda_{1}\right)}$ and hence EoS parameter is equal: $w=\frac{b_{1}^{2}}{3 \kappa^{2}\left(1+\lambda_{1}\right)}-1$. Also in this point, the scalar field potential dominates.

- $\mathbf{C}:(x, y, z, \lambda)=\left( \pm \frac{\sqrt{6}}{\sqrt{\kappa^{2}\left(1+2 \lambda_{1}\right)}}, 0,0, \lambda_{1}\right)(\lambda$ is constant). For this point $\mu=-\frac{3\left(1+\lambda_{1}\right)}{1+2 \lambda_{1}}$ and hence EoS parameter is $w=\frac{2\left(1+\lambda_{1}\right)}{1+2 \lambda_{1}}-1$. In this point the kinetic energy of the scalar field dominates.

- D: For this point one gets complicated expressions for $x$ and $y(x \neq 0, \quad y \neq 0)$, but $z=0$ and $\lambda=$ $\lambda_{1}=$ const Futhermore, one gets: $\mu=-\frac{x}{3 b_{2}}$.

If $\Lambda$ is not zero (we have to add one more equation $H^{\prime}=\mu H$ to the system (64)), then the point $\mathbf{A}$ does not change. However, it appears a new point $\mathbf{A} 1$ for which $H= \pm \frac{\kappa \sqrt{\Lambda}}{\sqrt{3}}$. This means that at least one more point with all of the variables not equal to zero exists. Following this analysis of critical points, it is straightforward to study the stability and attractor nature of cosmological solutions under discussion at finite and asymptotically.

Finally we want to stress that the presence of the Lagrange multiplier adds new critical points. Moreover, we can get a different value $w$ for the same potential by the modification of the constraint.

\section{CONCLUSIONS}

We have examined a string-inspired effective theory of gravity containing Gauss-Bonnet invariant interacting with a scalar field where a Lagrange multiplier is inserted into the action. The Lagrange multiplier term can be related to the process of string compactification that leads to the effective $4 \mathrm{D}$-action. Adopting a FRW metric, the corresponding cosmological Friedmann equations (7) and (8) can be used to define the effective potential and the Lagrange multiplier (and the function $\varepsilon$ which is the coupling to the Gauss-Bonnet term). Choosing a form for the metric and the scalar field, one can easily approach the cosmological reconstruction in a sort of inverse scattering approach. In general, the presence of the Lagrange multiplier helps in the generation of 
new cosmological solutions or in changing the features of some known solutions. This fact could be extremely relevant from an observational point of view because the Lagrange multipliers could be related to natural "priors" in order to discriminate among concurrent models. Specifically, relating cosmographic observed parameters as $\left\{H_{0}, q_{0}, j_{0}, s_{0}, \Omega_{M}, \Omega_{\Lambda}\right\}$ to some Lagrange multiplier could result a useful tool to restrict the range of models physically viable (see Ref.[2] and references therein for a detailed discussion on this point).

In the models that we have considered, the Lagrange multiplier can be interpreted as the presence of some dust fluid (matter without pressure) that affects the evolution of the cosmological system. In this case, the Lagrange multiplier can help to realize the transitions between matter dominance and dark energy era (and vice-versa) without imposing unnatural fine-tunings. The detailed study of the theory (2) with the exponential choice for the potential, the Lagrangian multiplier and the function $\varepsilon$ has been presented. Two forms of scalar field: $\varphi \sim t$ and $\varphi \sim \ln t$ are discussed. The comparison with the case of no Lagrange multiplier is considered. The presence of the Lagrange multiplier gives rise to new cosmological solutions due to the change in the dynamical system. For example, in the case of the scalar field with the logarithm form in time, the theory provides only one cosmological solution. In presence of the Lagrange multiplier, the number of cosmological solutions with different features increase. In particular, (ghost-free) phantom cosmology with canonical scalar easily emerges as solution. However, the situation can be more complicated since the Hubble rate $H$ is not necessarily a monotonic function of time. In some phantom models, dark energy grows with time and so does $H$ at late times. On the other hand, $H$ can decrease in matter-dominated eras so non-monotonic behaviors have to be considered. Above we gave also an example in this sense.

The extension to other modified gravities (teleparallel, Horava-Lifshitz, $F(G)$, non-local, etc [3]) via the introduction of the Lagrange multiplier can be easily accomplished in this framework. These topics will be discussed elsewhere.

\section{Appendix A: The Lagrange Multiplier Method and Noether Symmetries}

A more detailed discussion on the Lagrange Multiplier Method is necessary. A Lagrange multiplier is not introduced ad hoc in the dynamics but it is related to constraints of the theory related to symmetries and conservation laws. Here we will sketch the Noether Symmetry Approach (see Ref.[18] for a recent review) showing that the reduction of dynamics induced by the Lagrange multipliers is always related to the search for symmetries [28, 29]. In particular, the form of the Lagrange multiplier derives from conservation laws and then it has always a physical meaning.
We have used a FRW metric, so that the Einstein Eqs. (17) and (8) reduce to ordinary differential equations. The same equations can be derived from the action (2) where the Lagrangian becomes point-like after introducing in it a FRW metric. The configuration space is a minisuperspace where the Lagrangian coordinates are the scale factor $a$ and the scalar field $\varphi$, with the velocities $\dot{a}, \dot{\varphi}[18]$. In this case, the dimension of the space is 2 but there are cases in which the minisuperspace dimension can be larger (for instance a Bianchi universe with anisotropies 30] or dynamical systems with more than one scalar field [31]).

With these considerations in mind, let $\mathcal{L}\left(q^{i}, \dot{q}^{i}\right)$ be a Lagrangian, independent of time and nondegenerate, i.e.

$$
\frac{\partial \mathcal{L}}{\partial t}=0 ; \quad \operatorname{det} H_{i j} \stackrel{\operatorname{def}}{=} \operatorname{det}\left\|\frac{\partial^{2} \mathcal{L}}{\partial \dot{q}^{i} \partial \dot{q}^{j}}\right\| \neq 0,
$$

where det $H_{i j}$ is the Hessian determinant. In standard problems of analytical mechanics $\mathcal{L}$ has the form

$$
\mathcal{L}=T(\mathbf{q}, \dot{\mathbf{q}})-V(\mathbf{q})
$$

where $T$ and $V$ are the kinetic and potential energy. $T$ is a positive definite quadratic form in $\dot{\mathbf{q}}$. Associated with $\mathcal{L}$ is the energy function

$$
E_{\mathcal{L}} \equiv \frac{\partial \mathcal{L}}{\partial \dot{q}^{i}} \dot{q}^{i}-\mathcal{L}
$$

which is the total energy $T+V$. It is worth noticing that $\mathcal{L}$ can be more complicated than $\left(\mathrm{A2}\right.$ and $E_{\mathcal{L}}$ is a constant of the motion called "energy" also in general cases. In the Lagrangian formalism, we have to consider only transformations which are point-transformations. Any invertible and smooth transformation of the positions $Q^{i}=Q^{i}(\mathbf{q})$ induces a transformation of the velocities of the form

$$
\dot{Q}^{i}(\mathbf{q})=\frac{\partial Q^{i}}{\partial q^{j}} \dot{q}^{j}
$$

The matrix $\mathcal{J}=\left\|\partial Q^{i} / \partial q^{j}\right\|$ is the Jacobian of the transformation on the positions and it is assumed to be nonzero. The Jacobian $\mathcal{J}^{\prime}$ of the induced transformation is easily derived and $\mathcal{J} \neq 0 \rightarrow \mathcal{J}^{\prime} \neq 0$. A point transformation $Q^{i}=Q^{i}(\mathbf{q})$ can depend on one (or more than one) parameter. Let us assume that a point transformation depends on a parameter $\epsilon$, i.e. $Q^{i}=Q^{i}(\mathbf{q}, \epsilon)$, and that it gives rise to a one-parameter Lie group. For infinitesimal values of $\epsilon$, the transformation is then generated by a vector field: for instance, as well known, $\partial / \partial x$ represents a translation along $x$ axis, $x(\partial / \partial y)-y(\partial / \partial x)$ is a rotation around $z$ axis and so on. In general, an infinitesimal point transformation is represented by a generic vector field on $Q$

$$
\mathbf{X}=\alpha^{i}(\mathbf{q}) \frac{\partial}{\partial q^{i}}
$$


The induced transformation (A4), considering also velocities, is then represented by

$$
\mathbf{X}^{c}=\alpha^{i}(\mathbf{q}) \frac{\partial}{\partial q^{i}}+\left(\frac{d}{d t} \alpha^{i}(\mathbf{q})\right) \frac{\partial}{\partial \dot{q}^{j}} .
$$

$\mathbf{X}^{c}$ is called the "complete lift" of $\mathbf{X}[20]$. From now on, we will drop the suffix ${ }^{c}$ but clearly we refer to a complete lift. A function $f(\mathbf{q}, \dot{\mathbf{q}})$ is invariant under a transformation represented by $\mathbf{X}$ if

$$
L_{\mathbf{X}} f \stackrel{\text { def }}{=} \alpha^{i}(\mathbf{q}) \frac{\partial f}{\partial q^{i}}+\left(\frac{d}{d t} \alpha^{i}(\mathbf{q})\right) \frac{\partial f}{\partial \dot{q}^{j}}=0,
$$

where $L_{\mathbf{X}} f$ is the Lie derivative of $f$. In particular, if $L_{\mathbf{X}} \mathcal{L}=0, \mathbf{X}$ is said to be a symmetry for the dynamics derived by $\mathcal{L}$.

Let us consider now a Lagrangian $\mathcal{L}$ and its EulerLagrange equations

$$
\frac{d}{d t} \frac{\partial \mathcal{L}}{\partial \dot{q}^{j}}-\frac{\partial \mathcal{L}}{\partial q^{j}}=0 .
$$

Let us consider also a vector field of the form (A6). Contracting (A8) with the $\alpha^{i}$ 's gives

$$
\alpha^{j}\left(\frac{d}{d t} \frac{\partial \mathcal{L}}{\partial \dot{q}^{j}}-\frac{\partial \mathcal{L}}{\partial q^{j}}\right)=0 .
$$

Being

$$
\alpha^{j} \frac{d}{d t} \frac{\partial \mathcal{L}}{\partial \dot{q}^{j}}=\frac{d}{d t}\left(\alpha^{j} \frac{\partial \mathcal{L}}{\partial \dot{q}^{j}}\right)-\left(\frac{d \alpha^{j}}{d t}\right) \frac{\partial \mathcal{L}}{\partial \dot{q}^{j}},
$$

from (A9), we obtain

$$
\frac{d}{d t}\left(\alpha^{i} \frac{\partial \mathcal{L}}{\partial \dot{q}^{i}}\right)=L_{\mathbf{X}} \mathcal{L}
$$

The immediate consequence is the Noether Theorem: If $L_{\mathbf{X}} \mathcal{L}=0$, then the function

$$
\Sigma_{0}=\alpha^{i} \frac{\partial \mathcal{L}}{\partial \dot{q}^{i}},
$$

is a constant of motion.

Eq. A12 can be expressed independently of coordinates as a contraction of $\mathbf{X}$ with Cartan one-form

$$
\theta_{\mathcal{L}} \stackrel{\text { def }}{=} \frac{\partial \mathcal{L}}{\partial \dot{q}^{i}} d q^{i}
$$

For a generic vector field $\mathbf{Y}=y^{i} \partial / \partial x^{i}$, and one-form $\beta=\beta_{i} d x^{i}$, we have by definition $i_{\mathbf{Y}} \beta=y^{i} \beta_{i}$. Thus Eq. A12 can be written as

$$
i_{\mathbf{X}} \theta_{\mathcal{L}}=\Sigma_{0}
$$

Under a point-transformation, the vector field $\mathbf{X}$ becomes

$$
\mathbf{X}^{\prime}=\left(i_{\mathbf{X}} d Q^{k}\right) \frac{\partial}{\partial Q^{k}}+\left(\frac{d}{d t}\left(i_{x} d Q^{k}\right)\right) \frac{\partial}{\partial \dot{Q}^{k}}
$$

We see that $\mathbf{X}^{\prime}$ is still the lift of a vector field defined on the "positions" space only. If $\mathbf{X}$ is a symmetry and we choose a point transformation in such a way that

$$
i_{\mathbf{X}} d Q^{1}=1 ; \quad i_{\mathbf{X}} d Q^{i}=0 \quad i \neq 1,
$$

we get

$$
\mathbf{X}^{\prime}=\frac{\partial}{\partial Q^{1}} ; \quad \frac{\partial \mathcal{L}}{\partial Q^{1}}=0
$$

Thus $Q^{1}$ is a cyclic coordinate and the dynamics can be reduced according to well known procedures [20, 21]. The change of coordinates defined by (A16) is not unique. Usually a clever choice is very important. However, more than one symmetry can exist and the degree of dynamical reduction depends on the number of symmetries [18].

Let us now assume that $\mathcal{L}$ is a canonical Lagrangian (e.g. of the form (A2)). As $\mathbf{X}$ is of the form (A15), $L_{\mathbf{X}} \mathcal{L}$ will be a homogeneous polynomial of second degree in the velocities plus a inhomogeneous term in the $q^{i}$. Since such a polynomial has to be identically zero, each coefficient must be independently zero. If $n$ is the dimension of the configuration space, we get $\{1+n(n+1) / 2\}$ partial differential equations (PDE). The system is overdetermined, therefore, if a solution exists, it will be expressed in terms of integration constants instead of boundary conditions. Usually, $\mathcal{L}$ is fixed and one can ask for solutions of a specific PDE system. In particular, a Lagrangian containing some undefined functions (e.g. couplings $\omega(\varphi), \varepsilon(\varphi)$ and potentials $V(\varphi))$ is a class of $L a-$ grangians where the single element is determined by the solution of the PDE systems. In other words, the Noether symmetry selects the functions which assign the model.

The above discussion shows that, given a dynamical system, it is always possible to search for Noether symmetries. If they exist, the dynamics can be specified and reduced by a change of variables since one (or more than one) coordinate becomes cyclic. If we are able to integrate such a new dynamics, the problem is to invert the solution in order to get the evolution in the previous variables [28, 29]. Conversely, the existence of Noether symmetries is able to select the form of scalar field potentials and couplings. In other words, given a class of models, the symmetries are able to select some of them, which, in principle, are of physical interest.

The Lagrange multiplier method is related to this approach. In other words, imposing the existence of Lagrange multipliers means to select models where symmetries exist because the Lagrangian functions become canonical. The example below shows the strict relation between the Lagrange multipliers and the Noether symmetries.

Let us consider the simple case of $f(R)$ gravity defined by the action

$$
S=\int d^{4} x \sqrt{-g} f(R),
$$

where, as usual, $R$ is the Ricci scalar. This case is very interesting since not only the coupling and the potential 
are unspecified but the whole Lagrangian is not given a priori. Imposing, as above, the FRW metric, we can write

$$
S=\int \mathcal{L}(a, \dot{a}, R, \dot{R}) d t,
$$

considering $a$ and $R$ as canonical variables. Such a position is arbitrary since $R$ depends on $a, \dot{a}$ and $\ddot{a}$ so (A19) is not a true canonical Lagrangian. To remove this ambiguity, one can use a Lagrange multiplier $\lambda$ and search for Noether symmetry related to it. We have

$$
\begin{aligned}
S & =2 \pi^{2} \int d t \times \\
& \times\left\{f(R) a^{3}-\lambda\left[R+6\left(\frac{\ddot{a}}{a}+\frac{\dot{a}^{2}}{a^{2}}+\frac{k}{a^{2}}\right)\right]\right\} .
\end{aligned}
$$

To determine $\lambda$, we have to vary the action with respect to $R$, that is

$$
a^{3} \frac{d f(R)}{d R} \delta R-\lambda \delta R=0,
$$

from which we get, like in Sec.II, the functional form of the Lagrange multiplier, that is

$$
\lambda=a^{3} f^{\prime}(R) .
$$

Substituting into A20 and integrating by parts, we obtain the point-like Lagrangian

$$
\begin{aligned}
\mathcal{L} & =a^{3}\left[f(R)-R f^{\prime}(R)\right]+6 \dot{a}^{2} a f^{\prime}(R)+ \\
& +6 a^{2} \dot{a} \dot{R} f^{\prime \prime}(R)-a k f^{\prime}(R) .
\end{aligned}
$$

Then the cosmological equations of motion are

$$
\begin{aligned}
& \left(\frac{\ddot{a}}{a}\right) f(R)^{\prime}+2\left(\frac{\dot{a}}{a}\right) f(R)^{\prime \prime} \dot{R}+ \\
+ & f(R)^{\prime \prime} \ddot{R}+f(R)^{\prime \prime \prime} \dot{R}^{2}-\frac{1}{2}\left[R f(R)^{\prime}+f(R)\right]=0,
\end{aligned}
$$

and

$$
R=-6\left(\frac{\ddot{a}}{a}+\frac{\dot{a}^{2}}{a^{2}}+\frac{k}{a^{2}}\right),
$$

where, as above, the Lagrange multiplier gives one of the equations of motion. The energy constraint is

$$
\begin{aligned}
& 6 \dot{a}^{2} a f^{\prime}(R)-a^{3}\left[f(R)-R f^{\prime}(R)\right]+ \\
+ & 6 a^{2} \dot{a} \dot{R} f^{\prime \prime}(R)+a k f^{\prime}(R)=0 .
\end{aligned}
$$

The symmetry generator is defined on the tangent bundle $T Q(a, \dot{a}, R, \dot{R})$ and it is

$$
\mathbf{X}=\alpha(a, R) \frac{\partial}{\partial a}+\beta(a, R) \frac{\partial}{\partial R}+\frac{d \alpha}{d t} \frac{\partial}{\partial \dot{a}}+\frac{d \beta}{d t} \frac{\partial}{\partial \dot{R}},
$$

while the Noether condition $L_{\mathbf{X}} \mathcal{L}=0$ produces the system

$$
f^{\prime}(R)\left[\alpha+2 a \frac{\partial \alpha}{\partial a}\right]+a f^{\prime \prime}(R)\left[\beta+a \frac{\partial \beta}{\partial a}\right]=0,
$$

$$
a^{2} f^{\prime \prime}(R) \frac{\partial \alpha}{\partial R}=0
$$

$$
\begin{array}{cr}
2 f^{\prime}(R) \frac{\partial \alpha}{\partial R}+f^{\prime \prime}(R)\left[2 \alpha+a \frac{\partial \alpha}{\partial a}+a \frac{\partial \beta}{\partial R}\right]+a \beta f^{\prime \prime \prime}(R)= \\
=0, & (\mathrm{~A} 30) \\
3 \alpha\left[f(R)-R f^{\prime}(R)\right]-a \beta R f^{\prime \prime}(R)=0, & (\mathrm{~A} 31) \\
\alpha f^{\prime}(R)+a \beta f^{\prime \prime}(R)=0 . & \text { (A32) }
\end{array}
$$

From (A29), we have that $\alpha$ is a function of $a$ only, if we want to avoid trivial cases (i.e. we want $f^{\prime \prime}(R) \neq 0$ ). The symmetry is given by the functions

$$
\alpha=\frac{\beta_{0}}{a}, \quad \beta=-2 \beta_{0} \frac{R}{a^{2}}, \quad f(R)=f_{0} R^{3 / 2},
$$

which solve the above system; $\beta_{0}$ and $f_{0}$ are integration constants. The new induced variables can be

$$
w=a^{2} R, \quad z=\frac{a^{2}}{2 \beta_{0}},
$$

from which the Lagrangian (A23) becomes

$$
\tilde{\mathcal{L}}=\frac{9}{2} \beta_{0} \frac{\dot{z} \dot{w}}{\sqrt{w}}-9 k \sqrt{w}-\frac{1}{2} \sqrt{w^{3}},
$$

which can be rewritten in the form

$$
\tilde{\mathcal{L}}=9 \beta_{0} \dot{z} \dot{y}-9 k y-\frac{1}{2} y^{3},
$$

using $y=\sqrt{w}$. Dynamics is then described from the equations

$$
\ddot{y}=0, \quad \text { from which } \dot{y}=\dot{y}_{0}=\Sigma_{0},
$$

$$
9 \beta_{0} \ddot{z}+9 k+\frac{3}{2} y^{2}=0,
$$

$$
9 \beta_{0} \dot{y} \dot{z}+9 k y+\frac{1}{2} y^{3}=0,
$$

whose solutions are

$$
\begin{gathered}
y(t)=\dot{y}_{0} t+y_{0}, \\
z(t)=c_{4} t^{4}+c_{3} t^{3}+c_{2} t^{2}+c_{1} t+c_{0},
\end{gathered}
$$

with

$$
\begin{aligned}
& c_{4}=-\frac{\dot{y}_{0}^{2}}{72 \beta_{0}}, \quad c_{3}=-\frac{\dot{y}_{0} y_{0}}{3 \beta_{0}}, \\
& c_{2}=-\frac{y_{0}^{2}}{12 \beta_{0}}-\frac{k}{2}, \quad c_{1}=\dot{z}_{0}, \quad c_{0}=z_{0} .
\end{aligned}
$$


The energy condition (A39) gives the relation among the initial data [29]. Going back to the physical variables, we have

$$
a(t)= \pm \sqrt{d_{4} t^{4}+d_{3} t^{3}+d_{2} t^{2}+d_{1} t+d_{0}},
$$

where the $d_{i}$ 's are the $c_{i}$ 's multiplied by $2 \beta_{0}$. The $R$ variable is actually the Lagrange multiplier which gives

$$
R=\frac{\left(\dot{y}_{0} t+y_{0}\right)^{2}}{d_{4} t^{4}+d_{3} t^{3}+d_{2} t^{2}+d_{1} t+d_{0}},
$$

and then the cosmological equations (A24), (A25) and (A26) are fully satisfied. In conclusion, by imposing the Lagrange multiplier we get a canonical dynamics and the consequent existence of the Noether symmetry allows its integration. In summary, the dynamical system results both canonical and integrable. More complicated cases are discussed in [18, 19].

\section{Acknowledgments.}

This work has been supported by project 2.1839.2011 of Min. of Education and Science (Russia) and LRSS project 224.2012 .2 (Russia). S.C. is supported by INFN (Iniziative specifiche NA12 and OG51). The work by SDO is supported in part by MICINN (Spain), project FIS2010-15640 and by AGAUR (Generalitat de Catalunya), contract 2009SGR-994.
[1] A. G. Riess et al. [Supernova Search Team Collaboration], Astron. J. 116, 1009 (1998), astro-ph/980520; S. Perlmutter et al. [Supernova Cosmology Project Collaboration], Nature 391, 51 (1998), astro-ph/9712212, M. Hicken, W. M. Wood-Vasey, S. Blondin, P. Challis, S. Jha, P. L. Kelly, A. Rest and R. P. Kirshner, Astrophys. J. 700, 1097 (2009), arXiv:0901.4804 [astro-ph]; E. Komatsu et al. [WMAP Collaboration], Astrophys. J. Suppl. 180, 330 (2009), arXiv:0803.0547 [astro-ph]; W. J. Percival et al. [SDSS Collaboration], Mon. Not. Roy. Astron. Soc. 401, 2148 (2010), arXiv:0907.1660 [astro-ph].

[2] K. Bamba, S. Capozziello, S. Nojiri and S. D. Odintsov, Astrophys. Space Sci. 342, 155 (2012). arXiv :1205.3421 [gr-qc].

[3] S. Capozziello, Int.J.Mod.Phys. D 11, 483 (2002) [arXiv: gr-qc/0201033;

S. 'i. Nojiri and S. D. Odintsov, eConf C 0602061 (2006) 06 [Int. J. Geom. Meth. Mod. Phys. 4, 115 (2007)], hep-th/0601213

S. Nojiri and S. D. Odintsov, Phys. Rept. 505, 59 (2011), arXiv:1011.0544 [gr-qc];

S. Capozziello and M. De Laurentis, Phys. Rept. 509, 167 (2011), arXiv:1108.6266 [gr-qc].

[4] S. Capozziello and V. Faraoni, Beyond Einstein gravity, Fundamental Theories of Physics, Vol. 170, Springer, New York (2010).

[5] S. Capozziello and M. De Laurentis, Annalen Phys. 524, 545 (2012).

[6] M. Kowalski et al. [Supernova Cosmology Project Collaboration], Astrophys. J. 686, 749 (2008), arXiv:0804.4142 [astro-ph].

[7] K. Nakamura et al. [Particle Data Group Collaboration], J. Phys. G 37, 075021 (2010).

[8] R. Amanullah, C. Lidman, D. Rubin, G. Aldering, P. Astier, K. Barbary, M. S. Burns and A. Conley et al., Astrophys. J. 716, 712 (2010), arXiv:1004.1711] [astroph].

[9] http://www.euclid-ec.org/

[10] I. Antoniadis, J. Rizos, K. Tamvakis, Nucl. Phys. B 415, 497 (1994), arXiv:hep-th/9305025.

P. Kanti, J. Rizos and K. Tamvakis, Phys. Rev. D 59, 083512 (1999), arXiv:gr-qc/9806085
[11] N. E. Mavromatos and J. Rizos, Phys. Rev. D 62, 124004 (2000), arXiv:hep-th/0008074; JHEP 0207, 045 (2002), arXiv:hep-th/0205099 Int. J. Mod. Phys. A 18, 57 (2003), arXiv:hep-th/0205299.

[12] M. Gasperini, M. Maggiore and G. Veneziano, Nucl. Phys. B 494, 315 (1997), arXiv:hep-th/9611039,

R. Brustein and R. Madden, Phys. Rev. D 57, 712 (1998), arXiv:hep-th/9708046,

D. A. Easson and R. H. Brandenberger, JHEP 9909, 003 (1999), arXiv:hep-th/9905175

C. Cartier, E. J. Copeland and R. Madden, JHEP 0001, 035 (2000), arXiv:hep-th/9910169

S. Tsujikawa, R. Brandenberger and F. Finelli, Phys. Rev. D 66, 083513 (2002), arXiv:hep-th/0207228.

[13] S. Nojiri, S. D. Odintsov, M. Sasaki, Phys. Rev. D 71, 123509 (2005), arXiv:hep-th/0504052.

[14] M. Sami, A. Toporensky, P. V. Tretjakov, S. Tsujikawa, Phys.Lett. B619, 193 (2005), hep-th/0504154.

G. Calcagni, S.i Tsujikawa, M Sami, Class.Quant.Grav. 22, 3977-4006 (2005), hep-th/0505193;

S. Nojiri, S. D. Odintsov, Phys. Lett. B631, 1 (2005), hep-th/0508049,

G. Cognola, E. Elizalde, S. Nojiri, S. D. Odintsov, S. Zerbini, Phys. Rev. D73, 084007 (2006), hep-th/0601008

T. Koivisto, D. F. Mota, Phys. Lett. B644, 104 (2007), astro-ph/0606078,

B. M. Leith and I. P. Neupane, JCAP 0705, 019 (2007), arXiv:hep-th/0702002

S. Nojiri, S. D. Odintsov and P. V. Tretyakov, Phys. Lett. B651, 224 (2007), arXiv:0704.2520 [hep-th];

T. Koivisto, D. F. Mota, Phys. Rev. D75, 023518 (2007), hep-th/0609155

E. Elizalde, S. Jhingan, S. Nojiri, S.D. Odintsov, M. Sami, I. Thongkool, Eur.Phys.J. C53, 447-457 (2008), arXiv:0705.1211 [hep-th];

K. Bamba, Z. K. Guo, N. Ohta, Prog. Theor. Phys. 118, 879 (2007), arXiv:0707.4334 [hep-th];

F. S.N. Lobo, arXiv:0807.1640 [gr-qc];

M.R. Setare, E.N. Saridakis, Phys.Lett. B670, 1-4 (2008), arXiv:0810.3296 [hep-th];

Christian G. Bohmer, Francisco S.N. Lobo, Phys.Rev. D79, 067504 (2009), arXiv:0902.2982 [gr-qc]; 
L. N. Granda, E. Loaiza, Int. J. Mod. Phys. D21, 1250002 (2012), arXiv:1111.2454 [hep-th];

L. N. Granda, E. Loaiza, JCAP 1209, 011 (2012), arXiv:1209.1137 [hep-th];

L.N. Granda, D.F. Jimenez, C. Sanchez, arXiv:1211.3457 [astro-ph.CO];

S. Nojiri, E.N. Saridakis, arXiv:1301.2686 [hep-th].

[15] S. Nojiri, S. D. Odintsov, M. Sami, Phys. Rev. D74, 046004 (2006), hep-th/0605039.

[16] E. A. Lim, I. Sawicki and A. Vikman, arXiv:1003.5751 [astro-ph.CO].

[17] C. Gao, Y. Gong, X. Wang and X. Chen, arXiv:1003.6056 [astro-ph.CO].

[18] S. Capozziello, M. De Laurentis and S. D. Odintsov, Eur. Phys. J. C 72, 2068 (2012), arXiv:1206.4842 [gr-qc].

[19] S. Capozziello, J. Matsumoto, S. Nojiri, S.D. Odintsov, Phys. Lett. B 693, 198 (2010), arXiv:1004.3691 [hep-th].

[20] V. I. Arnold, Mathematical Methods of Classical Mechanics, Springer-Verlag Berlin (1989).

[21] S. Capozziello, R. De Ritis, C. Rubano, P. Scudellaro, Riv.Nuovo Cim. 19, N4, 1 (1996).

[22] S. Nojiri and S. D. Odintsov, Phys. Rev. D 83 (2011) 023001 arXiv:1007.4856 [hep-th]]; J. Kluson, S. 'i. Nojiri and S. D. Odintsov, Phys. Lett. B 701, 117 (2011) arXiv:1104.4286 [hep-th]].

[23] Yi-Fu Cai, E.N. Saridakis, Class.Quant.Grav. 28, 035010 (2011), arXiv:1007.3204 [astro-ph.CO]; D. Saez-Gomez , Phys.Rev. D 85, 023009 (2012) , arXiv:1110.6033 [hep-th];
L.N. Granda, E. Loaiza, JCAP 1209, 011 (2012), arXiv:1209.1137 [hep-th];

A Cid, P. Labrana, Phys. Lett. B 717, 10 (2012), arXiv:1209.2339 [astro-ph.CO].

[24] Artyom V. Astashenok, Sergei D. Odintsov, Phys.Lett. B 718, 1194 (2013), arXiv:1211.1888 gr-qc];

[25] P. H. Frampton, K. J. Ludwick and R. J. Scherrer, Phys. Rev. D 84, 063003 (2011), arXiv:1106.4996 [astroph.CO];

I. Brevik, E. Elizalde, S. Nojiri and S. D. Odintsov, Phys. Rev. D 84, 103508 (2011), arXiv:1107.4642 [hep-th];

[26] P. H. Frampton, K. J. Ludwick, S. Nojiri, S. D. Odintsov and R. J. Scherrer, Phys. Lett. B 708, 204 (2012), arXiv:1108.0067 [hep-th];

[27] S. Nojiri, S. D. Odintsov and D. Saez-Gomez, arXiv:1108.0767 hep-th];

A. N. Makarenko, V. V. Obukhov and I. V. Kirnos, Astrophys.Space Sci. 343481 (2013), arXiv:1201.4742 [grqc]; D. Saez-Gomez, arXiv:1207.5472,

[28] S. Basilakos, M. Tsamparlis, A. Paliathanasis, Phys. Rev. D 83, 103512 (2011), arXiv:1104.2980 [astro-ph.CO].

[29] S. Capozziello, A. De Felice, JCAP 0808, 016 (2008), arXiv:0804.2163 [gr-qc].

[30] S. Capozziello, G. Marmo, C. Rubano, P. Scudellaro, Int. J. Mod. Phys. D 6, 491 (1997), arXiv: gr-qc/9606050.

[31] S. Capozziello, G. Lambiase, Grav. Cosmol. 6164 (2000), arXiv: gr-qc/0003048. 